\title{
Dephasing due to Nuclear Spins in Large-Amplitude Electric Dipole Spin Resonance
}

\author{
Stefano Chesi, ${ }^{1, *}$ Li-Ping Yang, ${ }^{1}$ and Daniel Loss ${ }^{2,3}$ \\ ${ }^{1}$ Beijing Computational Science Research Center, Beijing 100084, China \\ ${ }^{2}$ Department of Physics, University of Basel, Klingelbergstrasse 82, 4056 Basel, Switzerland \\ ${ }^{3}$ CEMS, RIKEN, Wako, Saitama 351-0198, Japan
}

(Received 27 August 2015; revised manuscript received 26 October 2015; published 12 February 2016)

\begin{abstract}
We analyze effects of the hyperfine interaction on electric dipole spin resonance when the amplitude of the quantum-dot motion becomes comparable or larger than the quantum dot's size. Away from the wellknown small-drive regime, the important role played by transverse nuclear fluctuations leads to a Gaussian decay with characteristic dependence on drive strength and detuning. A characterization of spin-flip gate fidelity, in the presence of such additional drive-dependent dephasing, shows that vanishingly small errors can still be achieved at sufficiently large amplitudes. Based on our theory, we analyze recent electric dipole spin resonance experiments relying on spin-orbit interactions or the slanting field of a micromagnet. We find that such experiments are already in a regime with significant effects of transverse nuclear fluctuations and the form of decay of the Rabi oscillations can be reproduced well by our theory.
\end{abstract}

DOI: 10.1103/PhysRevLett.116.066806

Introduction.-The interest in coherent manipulation of single electron spins has stimulated intense research efforts, leading to a great degree of control in a variety of nanostructures [1,2]. For electrons in quantum dots, electron spin resonance (ESR) was first demonstrated in Ref. [3]. However, full electric control of local spins might be a better strategy for complex architectures of many quantum dots, envisioned to realize quantum information processing [4]. Thus, electric dipole spin resonance (EDSR) was developed relying on either spin-orbit couplings $[5,6]$ or the inhomogeneous magnetic field induced by a micromagnet $[7,8]$. The effectiveness of EDSR is highlighted by recent experiments, which could demonstrate Rabi oscillations with frequencies larger than $100 \mathrm{MHz}$ for both approaches $[9,10]$. To further improve the performance of such spin manipulation schemes, it is important to characterize relevant dephasing mechanisms, and especially those which might become dominant at a strong electric drive. In fact, while representing the main limitation for accurate spin manipulation, dephasing is still poorly understood in the large-amplitude regime of EDSR.

As we will show, one of the reasons is that the strong drive induces profound modifications on how typical dephasing mechanisms affect EDSR, and here we will focus on hyperfine interactions, which are well known to play an important role in the electron spin dynamics of quantum dots. In particular, the ESR dephasing was successfully interpreted in terms of a static Overhauser field, with a variance of a few millitesla in GaAs [3]. The resulting power-law decay and a universal $\pi / 4$ phase shift of the Rabi oscillations were accurately verified [11], confirming the predominance of nuclear spins over other sources of dephasing. While EDSR experiments were also generally interpreted assuming a power-law decay, the expected $t^{-1 / 2}$ dependence is violated at the larger values of the drive $[9,10,12]$. Specifically, Ref. [10] has demonstrated striking deviations from the ESR behavior, including a crossover from power-law to Gaussian decay. It is also generally known that the electron motion, as well as the presence of the drive, can have substantial effects on spin dynamics and decoherence [13-17], which motivates us to provide a detailed characterization of EDSR dephasing induced by the hyperfine interaction, paying special attention to the regime of large amplitude (i.e., comparable to the quantum dot's size). Since a main objective behind trying to achieve faster Rabi frequencies is to decrease operation errors, we also establish the limitations on spin-flip gate fidelity imposed on EDSR by the hyperfine interaction. Finally, we compare our theory with EDSR experiments which, as we will discuss, have very recently entered the large-amplitude regime.

Model.-EDSR is induced by a driven periodic displacement of the quantum dot $\vec{R}(t)=\hat{\mathbf{e}}_{x} \delta R \sin \omega t$, which we take conventionally along $x$. For the time-dependent wave function $\psi(\vec{r}-\vec{R}(t))$, we assume harmonic confinement along the direction of motion (which is applicable to both nanowire and lateral quantum dots):

$$
|\psi(\vec{r})|^{2}=|\varphi(y, z)|^{2} \frac{1}{\sqrt{\pi} \delta x} e^{-x^{2} / \delta x^{2}}
$$

The spin dynamics can be described with the following Hamiltonian:

$$
H=\frac{\epsilon_{z}}{2} \sigma_{z}+\frac{\vec{b} \cdot \vec{\sigma}}{2} \sin \omega t+\sum_{i} \frac{A_{i}}{n_{0}}\left|\psi\left(\vec{r}_{i}-\vec{R}(t)\right)\right|^{2} \vec{\sigma} \cdot \vec{I}_{i},
$$


where the first term is the electron Zeeman coupling, with $\epsilon_{z}=g \mu_{B} B$ and $\vec{\sigma}$ being the Pauli matrices. The second term is the drive, for which we can generally assume $b \propto \delta R$, while other features (e.g., the direction of $\vec{b}$ ) depend on specific details of the spin-orbit coupling or magnetic gradient. The last term in Eq. (2) is the Fermi contact hyperfine interaction, where $n_{0}$ is the nuclear density. $\vec{I}_{i}$ is the spin operator of nucleus $i$, with position $\vec{r}_{i}$ and coupling $A_{i}$. The periodic time dependence of Eq. (2) is characterized by Fourier components $\psi_{m}(\vec{r})=$ $(\omega / 2 \pi) \int_{0}^{2 \pi / \omega}|\psi(\vec{r}-\vec{R}(t))|^{2} e^{-i m \omega t} d t$, of which only the static $(m=0)$ and resonant $(m= \pm 1)$ ones are of interest here. In fact, in a frame rotating at frequency $\omega \simeq \epsilon_{z} / \hbar$ and neglecting fast oscillating terms, the transformed Hamiltonian $H^{\prime}$ reads

$$
\begin{aligned}
H^{\prime} \simeq & \frac{\epsilon_{z}-\hbar \omega}{2} \sigma_{z}-\frac{1}{4}(\vec{b} \times \vec{\sigma})_{z} \\
& +\sum_{i} \frac{A_{i}}{n_{0}}\left[\psi_{0}\left(\vec{r}_{i}\right) \sigma_{z} I_{i, z}+i \psi_{1}\left(\vec{r}_{i}\right)\left(\vec{\sigma} \times \vec{I}_{i}\right)_{z}\right],
\end{aligned}
$$

where longitudinal and transverse fluctuations are controlled by $\psi_{0}(\vec{r})$ and $\psi_{1}(\vec{r})$, respectively. Without loss of generality, we restrict ourselves to the case $b_{x}=b_{z}=0$ [18]:

$$
H^{\prime}=\frac{\Delta}{2} \sigma_{z}+\frac{b}{4} \sigma_{x}+\frac{1}{2} \vec{h} \cdot \vec{\sigma},
$$

where $\Delta=\epsilon_{z}-\hbar \omega$ is the detuning and $\vec{h}$ is defined by the second line of Eq. (3).

Nuclear fluctuations.-On the relatively short time scales of the EDSR experiments, it is appropriate to describe $\vec{h}$ with a static random classical magnetic field. In the lab frame and for infinite-temperature nuclear spins, the variance of the Overhauser field is given by $\sigma^{2}=\sum_{i}\left(2 A_{i} / n_{0}\right)^{2}\left|\psi\left(\vec{r}_{i}\right)\right|^{4} I_{i}\left(I_{i}+1\right) / 3$. However, $\vec{h}$ is for a reference frame moving with the dot and rotating at frequency $\omega$. As a consequence, its statistical properties differ from the ones in the lab frame. We still have $\langle\vec{h}\rangle=0$, but Eq. (3) implies that $\left\langle h_{z}^{2}\right\rangle$ and $\left\langle h_{x, y}^{2}\right\rangle$ have an interesting dependence on the strength of the drive. For finite $\delta R$ and $|\psi(\vec{r})|^{2}$, as in Eq. (1), we can evaluate $\left\langle h_{z}^{2}\right\rangle$ and $\left\langle h_{x, y}^{2}\right\rangle$ as follows, in terms of hypergeometric functions:

$$
\begin{aligned}
& \delta h_{z}=\frac{\sqrt{\left\langle h_{z}^{2}\right\rangle}}{\sigma}=\sqrt{{ }_{p} F_{q}\left(\frac{1}{2}, \frac{1}{2} ; 1,1 ;-\frac{2 \delta R^{2}}{\delta x^{2}}\right)}, \\
& \delta h_{x y}=\frac{\sqrt{\left\langle h_{x, y}^{2}\right\rangle}}{\sigma}=\frac{1}{2} \frac{\delta R}{\delta x} \sqrt{{ }_{p} F_{q}\left(\frac{3}{2}, \frac{3}{2} ; 2,3 ;-\frac{2 \delta R^{2}}{\delta x^{2}}\right)} .
\end{aligned}
$$

In the above formulas, the only dependence is on $\delta R / \delta x$, i.e., the amplitude of motion relative to the width of the electron wave function. $\delta h_{z}$ and $\delta h_{x y}$ are plotted in Fig. 1(a), showing
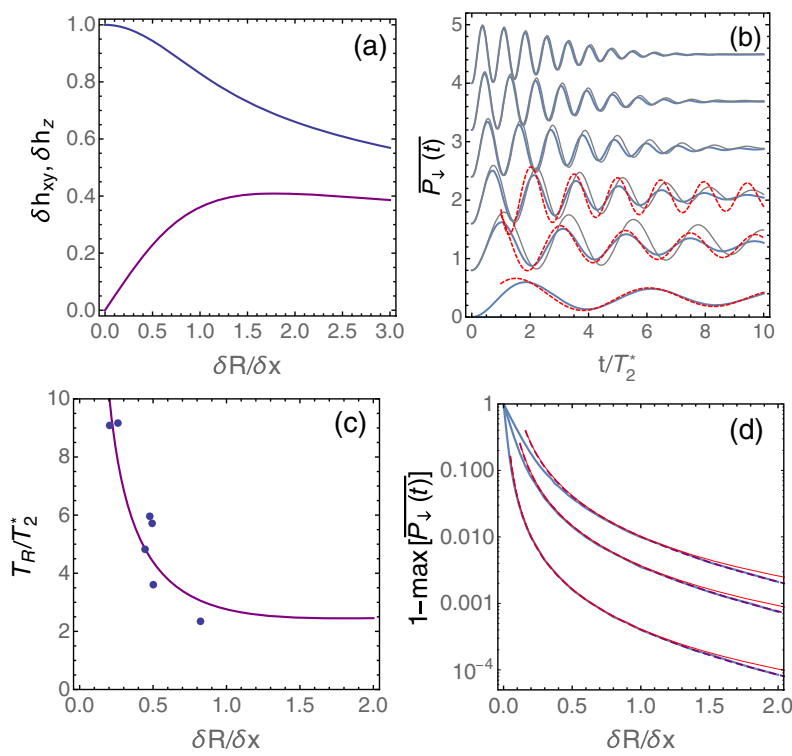

FIG. 1. (a) Plot of Eqs. (5) and (6), which characterize the longitudinal and transverse nuclear fluctuations (upper and lower curves, respectively). (b) Numerical result for the Rabi oscillations $\overline{P_{\downarrow}(t)}$, averaged over nuclear fluctuations (the thick blue curves). We used $\eta=0.05, \Delta=0$, and $\delta R / \delta x=0.1,0.2, \ldots, 0.6$ (from bottom to top). For clarity, the curves are shifted vertically. The red dashed curves are the asymptotic power-law decay of Ref. [11], valid when $\delta R / \delta x \lesssim \sqrt{2 \eta}=0.3$. The thin gray curves are from Eq. (9). (c) Plot of Eq. (12). Dots are decay times from Ref. [10], rescaled using $T_{2}^{*} \simeq 9$ ns and $\eta \simeq 0.09$. (d) Error in realizing a $\pi$ rotation, plotted as a function of $\delta R / \delta x$ at $\eta=$ $0.01,0.03,0.05$ (from bottom to top). Numerical results (the thick solid lines) are compared to the asymptotic result (the thick dashed lines) and the upper bound (the thin red lines) given in Eq. (13) [19]. The thin red lines also practically coincide with the ESR result, as seen by taking $\delta h_{z}=1$ and $\delta h_{x y}=0$ in Eq. (13).

that, for $\delta R \rightarrow 0$, only the longitudinal fluctuations survive. Therefore, in this limit one recovers the same behavior of ESR. At a finite $\delta R / \delta x$, the value of $\delta h_{z}$ is a decreasing function of amplitude, while the transverse fluctuations become nonzero and have a nonmonotonic dependence on $\delta R / \delta x$. Such transverse fluctuations can serve at $b=0$ as a driving term [13], and in this context they were previously discussed through an expansion at small $\delta R$ [14] or numerical evaluation [15]. However, both scenarios of EDSR (i.e., based on a micromagnet or spinorbit coupling) are in a physical regime distinct from Refs. [13-15] because $\delta h_{x y}$ is typically much smaller than the drive $b$. To see this, we notice that Eq. (6) implies $\delta h_{x y}<\frac{1}{2} \delta R / \delta x$; thus,

$$
\frac{h_{x, y}}{b} \sim \frac{\sigma \delta h_{x y}}{b}<\frac{\delta R / \delta x}{2 b / \sigma}=\frac{\eta}{2} \ll 1 .
$$

In Eq. (7) we defined the useful parameter $\eta=\sigma \delta R / b \delta x . \eta$ is approximately constant (since $b \propto \delta R$ ) and is typically 
small, according to our later estimates. Therefore, transverse nuclear fluctuations provide an additional dephasing mechanism which becomes progressively more important, until the maximum effect is reached at $\delta R / \delta x \simeq 1.8$. We will discuss how the effect of $\delta h_{x y}$ becomes dominant over $\delta h_{z}$ in a regime of a sufficiently strong EDSR drive, which was already realized in recent experiments [10].

Rabi oscillations.-We now use Eqs. (5) and (6) to perform a Gaussian average $\overline{P_{\downarrow}(t)}$ with respect to $\vec{h}$ of the spin-flip probability $P_{\downarrow}(t)$ :

$$
\begin{aligned}
P_{\downarrow}(t)= & \frac{\left(b / 2+h_{x}\right)^{2}+h_{y}^{2}}{\left(b / 2+h_{x}\right)^{2}+h_{y}^{2}+\left(\Delta+h_{z}\right)^{2}} \\
& \times \sin ^{2}\left(\frac{t}{2 \hbar} \sqrt{\left(b / 2+h_{x}\right)^{2}+h_{y}^{2}+\left(\Delta+h_{z}\right)^{2}}\right) .
\end{aligned}
$$

Although we cannot provide a general closed-form result, several relevant features can be explicitly characterized. In particular, at sufficiently large drive and detuning, we can neglect the components of $\vec{h}$ perpendicular to $(b / 2) \hat{\mathbf{e}}_{x}+\Delta \hat{\mathbf{e}}_{z}$ [see Eq. (4)] to obtain

$\overline{P_{\downarrow}(t)} \simeq \frac{b^{2} / 2}{b^{2}+4 \Delta^{2}}\left[1-e^{-\left(t / T_{R}\right)^{2}} \cos \left(\frac{t}{\hbar} \sqrt{b^{2} / 4+\Delta^{2}}\right)\right]$.

The Rabi decay time is

$$
T_{R}(\Delta)=\left(\frac{T_{2}^{*}}{\delta h_{x y}}\right) \times \sqrt{\frac{b^{2}+4 \Delta^{2}}{b^{2}+4 \Delta^{2}\left(\delta h_{z} / \delta h_{x y}\right)^{2}}},
$$

with $T_{2}^{*}=\sqrt{2} \hbar / \sigma$ being the typical inhomogeneous dephasing time associated with nuclear spins. Equation (9) implies a crossover between the ESR power-law decay at weak drive to the Gaussian decay of the strong-drive regime.

To exemplify this behavior, we first consider the resonant condition $(\Delta=0)$ when, besides $\delta R / \delta x$, the form of the decay is determined by $T_{2}^{*}$ and the coefficient $\eta$. An example of numerical results for $\overline{P_{\downarrow}(t)}$ is shown in Fig. 1(b), assuming $\eta=0.05$. We confirm that the ESR power-law decay $[3,11]$ is recovered when $\delta R / \delta x \rightarrow 0$, but significant deviations from this known dependence appear at a larger strength of the drive. In the large-drive limit, we find a Gaussian decay with no universal $\pi / 4$ phase shift, as Eq. (9) becomes an excellent approximation. This crossover to the strong-drive regime occurs when the effect of $h_{x}$ in Eq. (8) becomes dominant over $h_{z}$, i.e., $b h_{x} \gg h_{z}^{2}$. We estimate the typical values of $h_{x}, h_{z}$ using the limit of $\delta h_{x y}, \delta h_{z}$ at small $\delta R / \delta x$ (which is justified if $\eta \ll 1$ ), i.e., $h_{x} \sim \frac{1}{2} \sigma \delta R / \delta x$ and $h_{z} \sim \sigma$. This yields the condition

$$
\frac{\delta R}{\delta x} \gtrsim \sqrt{2 \eta},
$$

in good agreement with the numerical results of Fig. 1(b). Of special interest is the decay time scale:

$$
T_{R}(\Delta=0)=\frac{T_{2}^{*}}{\delta h_{x y}}
$$

which follows simply from Eq. (10) and is plotted in Fig. 1(c). The increasing strength of $\delta h_{x y}$ with the drive leads to a significantly faster decay, as also seen in the time domain results of Fig. 1(b). However, it is important to note that the fidelity of the $\pi$ rotation grows monotonically with $\delta R$, as shown in Fig. 1(d). For a quantitative analysis, we set $t=2 \pi \hbar / b$ in Eq. (8) and perform an expansion up to second order in $h$. After statistical averaging, we have

$$
1-\max \left[\overline{P_{\downarrow}(t)}\right] \simeq \frac{\eta^{2}\left(4 \delta h_{z}^{2}+\pi^{2} \delta h_{x y}^{2}\right)}{(\delta R / \delta x)^{2}}<\frac{4.1 \eta^{2}}{(\delta R / \delta x)^{2}} .
$$

Equation (13) includes a contribution proportional to $\delta h_{x y}^{2}$, but the additional dephasing from transverse fluctuations is more than compensated by the decrease of $\delta h_{z}^{2}$ and the faster Rabi frequency. Thus, Eq. (13) shows that it is always advantageous to apply a stronger drive and the effect of the hyperfine interaction on a $\pi$-rotation error can be reduced below any desired threshold with a sufficiently large $\delta R / \delta x[10]$.

Decay at finite detuning.-Considering a finite detuning yields further insight on the role of longitudinal and transverse nuclear fluctuations. The effect of $\Delta$ is illustrated in Fig. 2, where Fig. 2(c) confirms that Eq. (9) provides an excellent approximation in the strong-drive regime. A first consequence is that the Rabi oscillations approach the "chevron" pattern of Fig. 2(a). Furthermore, the decay time gets reduced at a finite $\Delta$, which is illustrated in Figs. 2(c) and $2(\mathrm{~d})$.

The dependence of $T_{R}$ on $\Delta$ has a simple physical explanation, as it can be traced to the difference in strength between the transverse and longitudinal nuclear fluctuations shown in Fig. 1(a). Since Eq. (4) implies that a finite detuning corresponds to a field along $z$ in the rotating frame, the relevant component of the nuclear fluctuations (i.e., along the total driving field) becomes a weighted average of $h_{x}$ and $h_{z}$. Since $\delta h_{z}>\delta h_{x y}$, the nuclear fluctuations gets enhanced by a finite detuning. As shown in Fig. 2(d), the dependence of $T_{R}$ on $\Delta$ is particularly pronounced at smaller values of $\delta R / \delta x$. This is natural, as the ratio $\delta h_{z} / \delta h_{x y}$ is large in this regime [see Fig. 1(a)], while the nuclear fluctuations become more isotropic at larger $\delta R / \delta x$. Thus, studying the dependence of $T_{R}$ on $\Delta$ allows one to explore how the relative strength of longitudinal and transverse nuclear fluctuations evolves with $\delta R / \delta x$. 

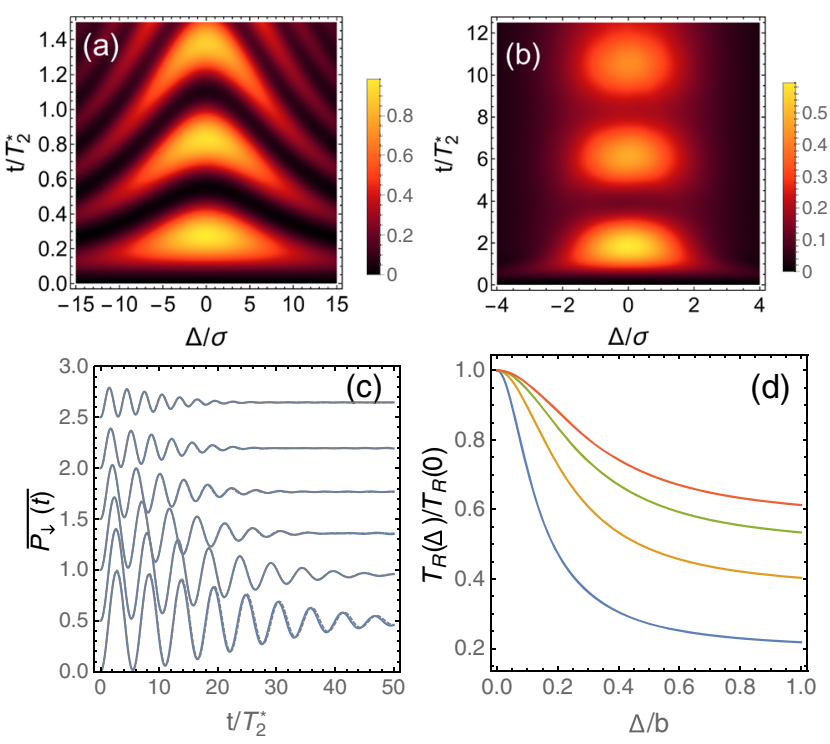

FIG. 2. Rabi oscillations at finite detuning. (a) and (b) represent $\delta R / \delta x=0.8$ and 0.1 , respectively. In both panels, $\eta=0.05$. (c) shows the line cuts of (a) at $\Delta / \sigma=0,2.5,5, \ldots, 12.5$ (from bottom to top). For clarity, the curves are shifted vertically. The numerical results are virtually indistinguishable from Eq. (9), plotted as gray dashed curves. In (d) we plot Eq. (10) as a function of detuning for several values of $\delta R / \delta x=0.4,0.8,1.2,1.6$ (from bottom to top).

Stationary limit.-We conclude our analysis of the Rabi oscillations by commenting briefly on the stationary limit $\overline{P_{\downarrow}(t \rightarrow \infty)}$, which is a useful quantity for estimating $\sigma$. Common methods rely either on the drive dependence at resonance (as done in the ESR experiment of Ref. [11]) or the linewidth (considering finite detunings). We find that, even if the Rabi oscillations are sensitively modified by transverse nuclear fluctuations, the effect on $\overline{P_{\downarrow}(\infty)}$ is negligible in the current experimental regime $\eta \ll 1$. A significant difference between ESR and EDSR only appears when $\eta \sim 1$ (for more details, see the Supplemental Material [20]). Therefore, methods to extract $\sigma$ from $\overline{P_{\downarrow}(\infty)}$ are still valid for large-amplitude EDSR.

Comparison to experiments. -We now discuss the application of our theory to available experimental data. In Fig. 3 we show an analysis of the data shown in Fig. 2(c) of Ref. [9], obtained from InSb nanowire dots with a strong spin-orbit interaction. As seen, our theory is able to reproduce well the Rabi oscillations and the fit yields values $\delta R / \delta x$ and $b$ that are consistent with $b \propto \delta R / \delta x$. For EDSR driven by the spin-orbit coupling, we have

$$
\eta=\frac{l_{\mathrm{SO}}}{2 \delta x} \frac{\sigma}{\epsilon_{z} \sin \theta}
$$

where $l_{\mathrm{SO}}$ is the spin-orbit length and $\theta$ the angle between the spin-orbit field and $\vec{B}$. Using $l_{\text {SO }}=200-300 \mathrm{~nm}$, $\delta x \simeq 10-20 \mathrm{~nm}$ [21], $g=41$, and $B=31.4 \mathrm{mT}$ [9] gives
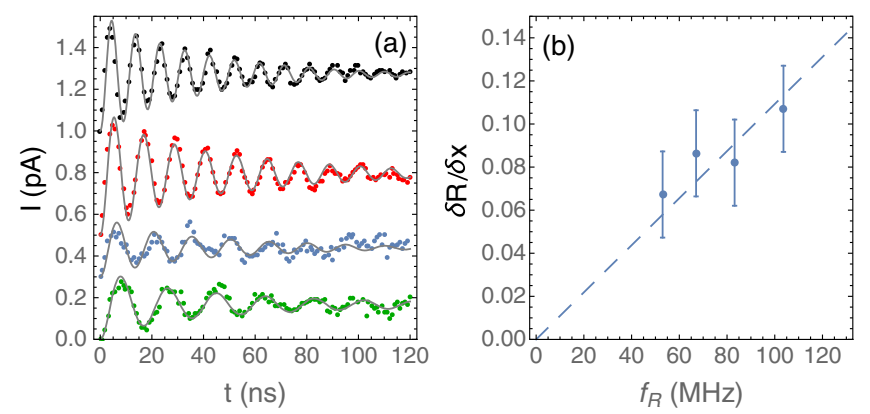

FIG. 3. (a) Fit of our theory to Rabi oscillations of Ref. [9]. For each curve, the fit parameters are $\delta R / \delta x$, a conversion factor to current, and $b=4 \pi \hbar f_{R}$, while $\sigma=0.22 \pm 0.03 \mu \mathrm{eV}$ is from the experiment [9]. (b) $\delta R / \delta x$ and $f_{R}$ obtained from the fits in (a). The error bars are from the uncertainty on $\sigma$. The dashed line is a fit to $\delta R / \delta x=C f_{R}$.

$\eta \simeq(0.015-0.04) / \sin \theta$. Figure 3(b) implies $\eta \sim 0.03$, which is consistent with this estimate.

While Fig. 3(b) has $\delta R / \delta x \lesssim 0.1$, we estimate that larger values of $\delta R / \delta x$ were achieved in a recent experiment on GaAs quantum dots [10]. There, the drive is based on a micromagnet for which numerical simulations give $b / \delta R \sim|g| \mu_{B} \times(1 \mathrm{mT} / \mathrm{nm}) \quad[10]$. Using the largest achieved Rabi frequency $f_{\max } \sim 120 \mathrm{MHz},|g| \simeq 0.4$, and $\delta x \sim 35-60 \mathrm{~nm}$ (corresponding to orbital energies $\sim 0.3-1 \mathrm{meV}$ ), we obtain $\delta R_{\max } / \delta x \sim 0.7-1.2$, which is relatively close to the condition at which $h_{x}$ fluctuations are most effective. On the other hand, the regime of motional narrowing $\delta R / \delta x \gtrsim 1.8$ [22] does not appear to be within reach of current experiments. For GaAs quantum dots $\sigma \sim|g| \mu_{B} \times(1-4 \mathrm{mT})[3,11,13,23]$, which allows us to estimate a typical range $\eta \sim 0.02-0.1$.

Several findings of Ref. [10] are in good agreement with our discussion, including the transition to a chevron pattern in the strong-drive regime [10]. As a result, Figs. 2(a) and 2(b) are remarkably similar to the corresponding panels of Ref. [10]. Furthermore, a crossover from power-law decay (for $b \lesssim|g| \mu_{B} \times 5 \mathrm{mT}$ ) to Gaussian decay (for $b \gtrsim|g| \mu_{B} \times$ $15 \mathrm{mT}$ ) was observed. The strength of the drive for such a crossover is compatible with Eq. (11), which can be rewritten as $b \gtrsim \sqrt{2 \sigma \delta x(b / \delta R)} \sim|g| \mu_{B} \times(8-20 \mathrm{mT})$, using the above estimates of $\sigma, \delta x$, and $b / \delta R$. We also show in Fig. 1(c) that Eq. (12) is able to reproduce the dependence of $T_{R}$ on the drive strength, with reasonable fit parameters for GaAs quantum dots $\left(T_{2}^{*} \simeq 9 \mathrm{~ns}, \eta \simeq 0.09\right)$.

Conclusion.-In conclusion, we have characterized the dephasing induced by the hyperfine interaction in largeamplitude EDSR, and we showed that transverse fluctuations of the Overhauser field are likely to play an important role in this regime, recently achieved experimentally. It should be mentioned that also other dephasing sources were suggested for EDSR, such as paramagnetic impurities, charge noise, and photon-assisted tunneling $[9,10,12]$. In the absence of clear evidence (or specific 
predictions) about these alternative mechanisms, our theory offers a further means to test whether nuclear spins are indeed the dominant effect, e.g., through a detailed analysis of $T_{R}$ as a function of both drive and detuning. In fact, it is unlikely that other types of dephasing would induce the same type of sensitive dependence on $\Delta$ discussed in relation to Eq. (10) and Fig. 2.

From a more general point of view, our study is helpful to assess the limitations to spin manipulation due to the quantum-dot motion and the nuclear-spin bath. These two aspects are unavoidable for EDSR based on III-V semiconductors, in contrast to ESR or spin manipulation based on group-IV materials [24,25]. Nuclear fluctuations, on the other hand, do not represent a fundamental obstacle to EDSR, since high-fidelity gates can be achieved at sufficiently large amplitude.

We thank W. A. Coish, T. Otsuka, P. Stano, and J. Yoneda for useful discussions. S. C. acknowledges funding from NSFC (Grant No. 11574025). D. L. acknowledges support from the Swiss NSF and NCCR QSIT.

*stefano.chesi@csrc.ac.cn

[1] R. Hanson, J. R. Petta, S. Tarucha, and L. M. K. Vandersypen, Rev. Mod. Phys. 79, 1217 (2007).

[2] D. D. Awschalom, L. C. Bassett, A. S. Dzurak, E. L. Hu, and J. R. Petta, Science 339, 1174 (2013).

[3] F. H. L. Koppens, C. Buizert, K. J. Tielrooij, I. T. Vink, K. C. Nowack, T. Meunier, L. P. Kouwenhoven, and L. M. Vandersypen, Nature (London) 442, 766 (2006).

[4] D. Loss and D. P. DiVincenzo, Phys. Rev. A 57, 120 (1998).

[5] V. N. Golovach, M. Borhani, and D. Loss, Phys. Rev. B 74, 165319 (2006).

[6] K. C. Nowack, F. H. L. Koppens, Y. V. Nazarov, and L. M. K. Vandersypen, Science 318, 1430 (2007).

[7] Y. Tokura, W. G. van der Wiel, T. Obata, and S. Tarucha, Phys. Rev. Lett. 96, 047202 (2006).

[8] M. Pioro-Ladrière, T. Obata, Y. Tokura, Y.-S. Shin, T. Kubo, K. Yoshida, T. Taniyama, and S. Tarucha, Nat. Phys. 4, 776 (2008).

[9] J. W. G. van den Berg, S. Nadj-Perge, V. S. Pribiag, S. R. Plissard, E. P. A. M. Bakkers, S. M. Frolov, and L. P. Kouwenhoven, Phys. Rev. Lett. 110, 066806 (2013).

[10] J. Yoneda, T. Otsuka, T. Nakajima, T. Takakura, T. Obata, M. Pioro-Ladrière, H. Lu, C. J. Palmstrøm, A. C. Gossard, and S. Tarucha, Phys. Rev. Lett. 113, 267601 (2014).
[11] F. H. L. Koppens, D. Klauser, W. A. Coish, K. C. Nowack, L. P. Kouwenhoven, D. Loss, and L. M. K. Vandersypen, Phys. Rev. Lett. 99, 106803 (2007).

[12] S. Nadj-Perge, S. M. Frolov, E. P. A. M. Bakkers, and L. P. Kouwenhoven, Nature (London) 468, 1084 (2010).

[13] E. A. Laird, C. Barthel, E. I. Rashba, C. M. Marcus, M. P. Hanson, and A. C. Gossard, Phys. Rev. Lett. 99, 246601 (2007).

[14] E. I. Rashba, Phys. Rev. B 78, 195302 (2008).

[15] G. Széchenyi and A. Pályi, Phys. Rev. B 89, 115409 (2014).

[16] C. Echeverría-Arrondo and E. Y. Sherman, Phys. Rev. B 87, 081410 (2013).

[17] J. Jing, P. Huang, and X. Hu, Phys. Rev. A 90, 022118 (2014).

[18] For an infinite-temperature nuclear bath, we can always choose the drive along $x$ in spin space due to the rotational invariance of the hyperfine coupling in the $x$-y plane; see Eq. (3). The strength of the drive is $b_{\perp}=\sqrt{b_{x}^{2}+b_{y}^{2}}=b$ if $b_{z}=0$. If $b_{z} \neq 0$, one should substitute $b \rightarrow b_{\perp}$ into Eq. (4) and in the rest of the Letter.

[19] For $\delta R / \delta x \lesssim 1$, the approximation of $1-\max \left[\overline{P_{\downarrow}(t)}\right]$ given in Eq. (13) is very close to its upper bound. However, at large values of $\delta R$, Eq. (13) gives $1-\max \left[\overline{P_{\downarrow}(t)}\right] \simeq$ $\sqrt{2 / \pi^{3}}\left(4+\pi^{2}\right) \eta^{2}(1.29+\ln \delta R / \delta x) /(\delta R / \delta x)^{3}, \quad$ which approaches zero faster than the upper bound.

[20] See the Supplemental Material at http://link.aps.org/ supplemental/10.1103/PhysRevLett.116.066806 for a more detailed analysis of the stationary value.

[21] S. Nadj-Perge, V. S. Pribiag, J. W. G. van den Berg, K. Zuo, S. R. Plissard, E. P. A. M. Bakkers, S. M. Frolov, and L. P. Kouwenhoven, Phys. Rev. Lett. 108, 166801 (2012).

[22] When $\delta R / \delta x \gg 1$, the large-amplitude motion induces an averaging of several independent nuclear configurations, separated by a distance $\sim \delta x[15]$. This in turn leads to a decrease of $\delta h_{x y}$.

[23] J. R. Petta, A. C. Johnson, J. M. Taylor, E. A. Laird, A. Yacoby, M. D. Lukin, C. M. Marcus, M. P. Hanson, and A. C. Gossard, Science 309, 2180 (2005).

[24] E. Kawakami, P. Scarlino, D. R. Ward, F. R. Braakman, D. E. Savage, M. G. Lagally, M. Friesen, S. N. Coppersmith, M. A. Eriksson, and L. M. K. Vandersypen, Nat. Nanotechnol. 9, 666 (2014).

[25] M. Veldhorst, J. C. C. Hwang, C. H. Yang, A. W. Leenstra, B. de Ronde, J. P. Dehollain, J. T. Muhonen, F. E. Hudson, K. M. Itoh, A. Morello, and A. S. Dzurak, Nat. Nanotechnol. 9, 981 (2014). 